\title{
Ultrastructures of the Golgi Body and Cell Surface in Cyanidioschyzon merolae
}

\author{
Tohru Okuwaki ${ }^{1}$, Hidenori Takahashi ${ }^{1}$, Ryuuichi Itoh ${ }^{1}$, Kyoko Toda ${ }^{1}$, \\ Tamotsu Kawazu' ${ }^{1}$ Haruko Kuroiwa ${ }^{2}$ and Tsuneyoshi Kuroiwa ${ }^{1}$ \\ ${ }^{1}$ Department of Biological Sciences, Graduate School of Science, \\ University of Tokyo, Hongo, Tokyo 113, Japan \\ ${ }^{2}$ Division of Biology, Kyoritsu Women's Junior College, Kanda, Tokyo 102, Japan
}

Accepted December 1, 1995

We are deeply interested in determining the minimum ultrastructural constituents of plant eukaryotic cells. Cyanidioschyzon merolae and Cyanidium caldarium are very small unicellular eukaryotes which range from $2 \mu \mathrm{m}$ to $6 \mu \mathrm{m}$ in diameter (De Luca et al. 1978, Merola et al. 1981). Each alga contains one cell nucleus, one mitochondrion and one chloroplast (De Luca et al. 1978, Merola et al. 1981). Merola et al. (1981) reported that C. merolae multiplied by binary division, while $C$. caldarium had 4 endospores, which indicated that $C$. caldarium multiplied by two consecutive binary divisions. Suzuki et al. (1992) reported that the cell nuclei in $C$. merolae and $C$. caldarium forma A had DNA contents of $8 \mathrm{Mbp}$ and $18 \mathrm{Mbp}$, respectively. We examined both $C$. merolae and $C$. caldarium using a serial thin-section electron microscopic technique after rapid freezing in liquid propane and a substitution fixation (Kuroiwa et al. 1994). The results showed that many basic organelles could be observed in both algae. However, while a Golgi body and a cell wall could be observed in $C$. caldarium cells, these could not be observed in C. merolae cells.

The aim of this study was to re-examine the existence of the Golgi body and cell surface in $C$. merolae using rapid freezing in liquid propane and a substitution fixation, and silver methenamine staining.

\section{Materials and methods}

C. merolae and C. caldarium RK-1 were generous gifts from Prof. Pinto (Napoli University, Italy) and Prof. Nagashima (Science University of Tokyo, Japan), respectively. They were cultured in Allen's medium (Allen 1959) under $\mathrm{CO}_{2}$ aeration at $40^{\circ} \mathrm{C}$ with a $12-\mathrm{hr}$ light/dark cycle at a light intensity of about 3,000lux. Division of $C$. merolae cells was synchronized by the method of Suzuki et al. (1994). C. merolae cells were collected in test tubes after the cells were slightly swelled in distilled water. Sample pellets were sandwiched between copper plates $(1 \mathrm{~mm} \times 4 \mathrm{~mm} \times 0.2 \mathrm{~mm})$, and put into cooled liquid propane at $-190^{\circ} \mathrm{C}$, which was replaced by acetone containing $1 \%$ osmium at $-85^{\circ} \mathrm{C}$. For conventional chemical fixation, the cells were fixed in $2 \%$ glutaraldehyde and $2 \% \mathrm{OsO}_{4}$ solution according to the standard method. Finally, all of the samples were embedded in Spurr resin. Thin sections were cut on an ultramicrotome with either a glass or diamond knife (Eiko En. Co., Kawasaki). The ultrathin serial sections were double-stained with 3\% uranyl acetate and 1\% lead citrate and observed under an electron microscope (JEOL 1200CX, Tokyo). In addition, the thin sections were stained with silver methenamine for electron microscopic demonstration of polysaccharide-like structures (Rambourg and Leblond 1967, Kuroiwa 1989). 
Results

\section{Golgi Apparatus}

In a previous report, we could not identify a typical Golgi body in $C$. merolae cells which were fixed under conventional chemical fixation or rapid freeze substitution fixation (Kuroiwa et al. 1994). In this study, we re-examined the fine structure of the cytoplasm in $C$. merolae cells fixed by rapid freezing. In $C$. merolae cells, chloroplast division, mitochondrial division and cell-nuclear division occur in that order during cell division (Kuroiwa et al. 1995). Fig. 1A shows a cell during the early stage of cell division. The dense material of the plastid-divising ring can be seen around the chloroplast (arrowhead in Fig. 1A). Golgi bodies appeared in the cytoplasm close to both poles of the cell nucleus (Fig. 1A). One Golgi body was cut at its periphery. The Golgi body was composed of transfer vesicles (small arrow in Fig. 1A), two Golgi cisternae (mid-size arrow in Fig. 1A) and secretory vesicles (large arrow in Fig. 1A). An association between the transfer vesicles and the nuclear membrane could often be seen (Fig. 1A). The secretory vesicles were distributed throughout the entire cytoplasm (large arrows in Fig. 1A).

Fig. 1B-D shows two serial thin-sections of a cell containing a dividing chloroplast. As chloroplast division progressed, the dense materials of the plastid-dividing ring and microbodies appeared at the bridge of the dividing chloroplast (Fig. 1B, C). Golgi bodies remained near both poles of the cell nucleus. At a higher magnification, the Golgi body was composed of transfer vesicles (small arrow in Fig. 1D), a Golgi cisterna (mid-size arrow in Fig. 1D) and secretory vesicles (large arrows in Fig. 1D). After cytokinesis, the Golgi bodies were separated into the daughter cells (data not shown). Based on these results, we concluded that $C$. merolae cells contain one Golgi body.

\section{Cell surface}

Since $C$. merolae cells maintain regular shapes, such as football-, peanut- and delta-shaped structures, it was expected that there was a special structure on the cell surface. However, when C. merolae and $C$. caldarium RK-1 were mixed, fixed and stained under the same conditions to avoid obtaining faulty results due to technical differences, a typical cell wall was seen in $C$. caldarium RK-1, but not in C. merolae as described previously (Fig. 2A, Kuroiwa et al. 1994). When we re-examined the fine structures of these cells, we noticed that there were many filamentous structures on the exterior surface of the cell membrane (large arrow in Fig. 2A). The cell membrane was coated with an electron-dense thin layer from which the filaments approximately $100 \mathrm{~nm}$ in length and $8 \mathrm{~nm}$ in diameter protruded. Such filamentous structurs did not appear on the surface of a cell wall of C. caldarium RK-1 (small arrows in Fig. 2A).

To elucidate whether or not the filaments in $C$. merolae cells contained polysaccharide, as do those in other plants, we stained $C$. merolae cells with silver methenamine for comparison with $C$. caldarium RK-1. Silver methenamine accumulated in a thin layer on the exterior surface of $C$. merolae cells (large arrow in Fig. 2B) and on the cell wall of $C$. caldarium RK1 (small arrows in Fig. 2B). In addition, these dense materials appeared on starch grains in

Fig. 1. Electron micrographs of Cyanidioschyzon merolae cells which contain a cell nucleus (n), chloroplasts (c), mitochondria $(\mathrm{m})$, a microbody $(\mathrm{mb})$ and Golgi bodies (asterisks) during the early stage of cell division (A) and the middle stage of cell division (B-D). Golgi bodies were composed of transfer vesicles (small arrows in A and D), one to two cisternae (mid-size arrow in $\mathrm{A}$ and $\mathrm{D}$ ) and secretory vesicles (large arrows in $\mathrm{A}$ and $\mathrm{D}$ ). $\mathrm{B}$ and $\mathrm{C}$ were serial thin sections. (D) is high-magnification image of the area in (C) labeled with asterisks. The appearance of the dense material of a plastid-dividing ring (arrowheads in A and B) suggests cell division. Scale bars represent $1 \mu \mathrm{m}(\mathrm{A}-\mathrm{C})$ and $0.1 \mu \mathrm{m}$ (D). 


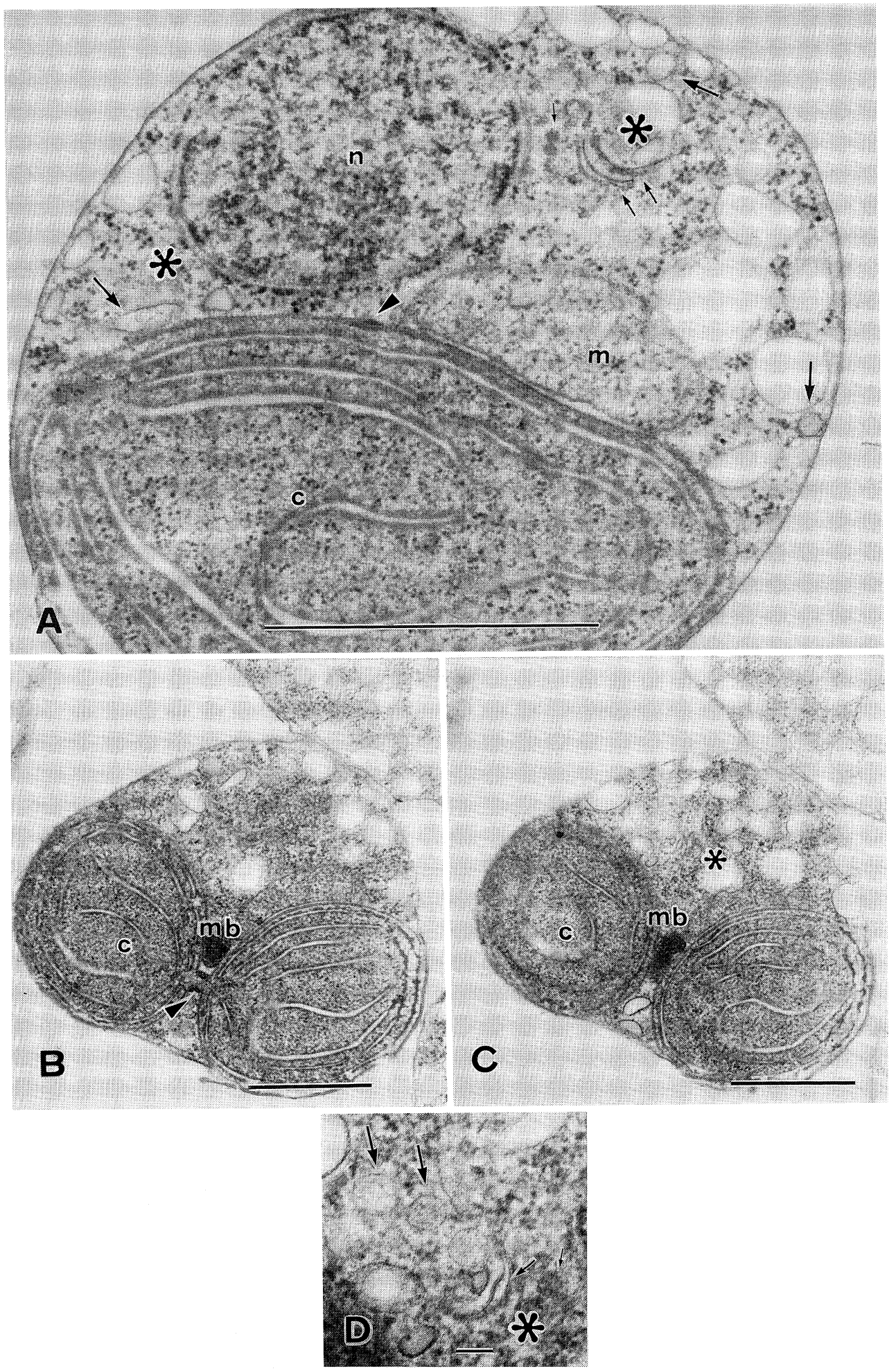

Fig. 1 


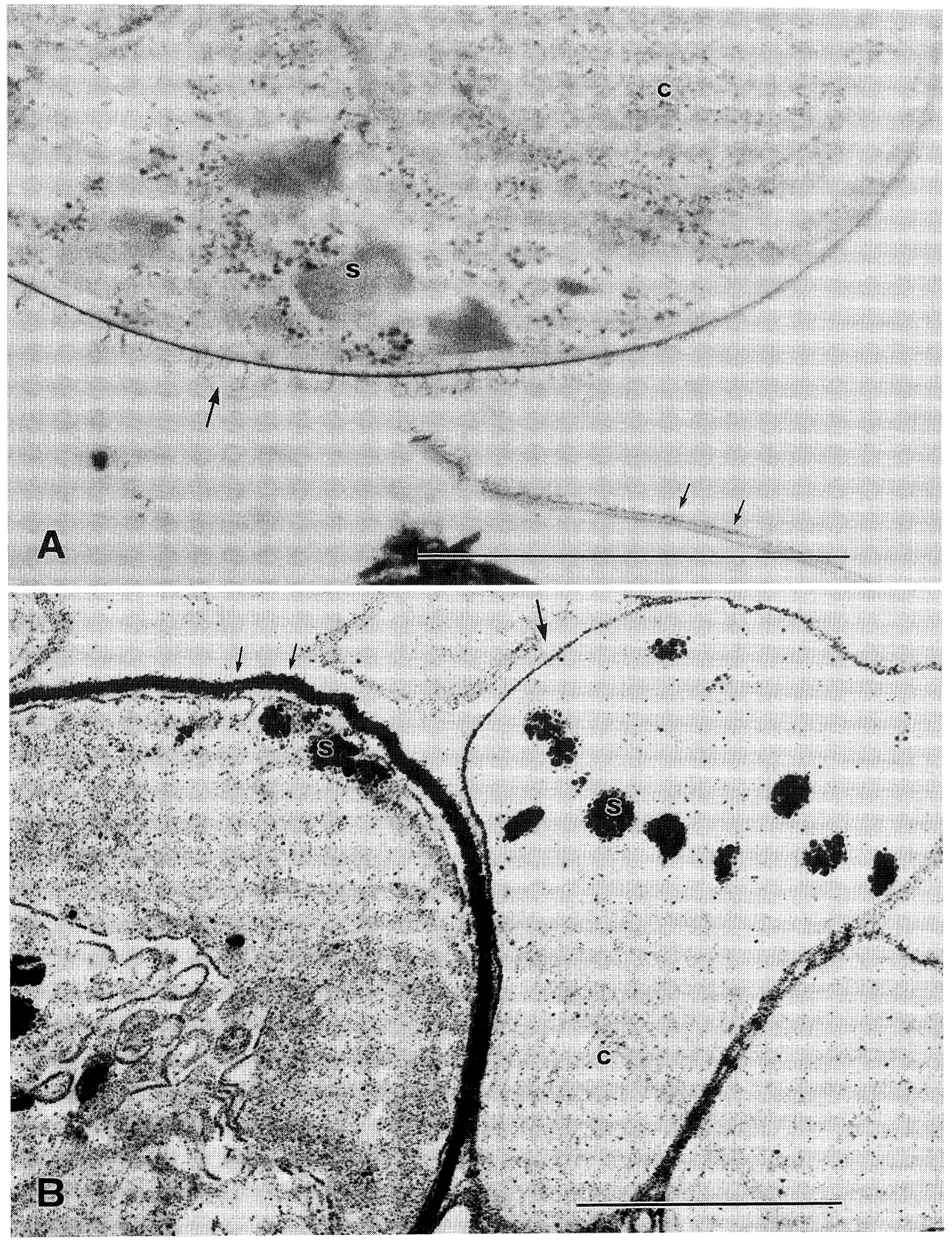

Fig. 2. Electron micrographs of cells which contained chloroplasts (c) and starch grains (s in $\mathrm{A}$ and B) during interphase cells in Cyanidioschyzon merolae and Cyanidium caldarium RK-1 before (A) and after treatment with silver methenamine (B). C. merolae cells were covered with a filamentous layer which protruded from an electron-dense layer (large arrow in A), while the cell wall of a broken $C$. caldarium cell was observed (small arrows in A). After staining with silver methenamine, dense materials appeared on the exterior surface of the cell membrane in $C$. merolae, the cell wall in C. caldarium RK-1 and starch grains in both cells. Scale bars represent $1 \mu \mathrm{m}(\mathrm{A}$ and $\mathrm{B})$. 
both cells (s in Fig. 2B). These results suggest that the cell surface of $C$. merolae is different from the cell wall of general algae and plants and that $C$. merolae cells maintain their shape using a specific filamentous layer.

\section{Discussion}

\section{Golgi Apparatus}

Broadwater and Scott (1994) summarized the characteristics of cells in various unicellular red algae. Golgi bodies are observed in many red algae, as well as in other eukaryotes. Red algae such as Porphyridium purpureum and Glaucosphaera vacuolata have typical Golgi bodies. Each cell contains several Golgi bodies which have more than 8 Golgi cisternae, while the primitive red algae, Galdieria sulphuraria (Ueda and Yokochi 1981) and C. caldarium forma A contain a few Golgi bodies (Kuroiwa et al. 1994). Golgi cisternae in G. sulphuraria and $C$. caldarium contain approximately 5 and 3 Golgi cisternae, respectively. In this study, $C$. merolae cells had a single Golgi body which was composed of one or two Golgi cisternae, secretory vesicles and transfer vesicles. However, a trans-Golgi network was not observed. The results suggest that Golgi bodies in $C$. merolae have a simpler architecture than those in other eukaryotes. The Golgi apparatus in C. merolae is arranged around the periphery of the cell nucleus. Since the transfer vesicles could be seen between the cell nucleus and Golgi cisternae, the Golgi bodies in C. merolae might differ from those in G. sulphuraria (Ueda and Yokochi 1981) and be similar to those in Dixoniella grisea, which present a third type of Golgi association (Broadwater and Scott 1994).

Since $C$. merolae cells in this study contained only one Golgi body, suplication of the Golgi body can be studied in detail during the mitotic cycle.

\section{Cell surface}

All non-controversial unicellular members of the Porphyridiales (Chapman 1974) and G. vacuolata (Richardson and Brown 1970) lack a cell wall, and instead have a mucilaginous sheath, the thickness of which varies with the culture conditions. In a previous paper, we reported that $C$. caldaium cells have a cell wall, while $C$. merolae cells do not. The present experiments confirmed these results. The surface of the cell membrane in $C$. merolae had a filamentous layer with a structure similar to those in the red alga, $R$. violacea and the giant amoeba, Peromyxa carolinensis (Giese 1979), which may move using these filamentous structures. These filaments may contain actin. However, althouth $C$. merolae contains an actin gene (Takahashi et al. 1995), the surface of the cells was not stained with phalloidin (Suzuki et al. 1995). These results suggest that the filaments at the exterior surface of the cell membrane do not contain actin.

After staining with silver methenamine, the electron-dense materials were seen on the cell wall of $C$. caldarium cells and on starch grains in both $C$. caldarium and $C$. merolae cells. These results suggest that the components of the cell wall in $C$. caldarium are similar to those of higher organisms.

Although $C$. merolae and C. caldarium live together in acidic hot springs, $C$. merolae does not have a cell wall while $C$. caldarium have a hard cell wall. Adaptation may play a role in this difference.

\section{Summary}

The presence of a Golgi body and a cell wall in Cyanidioschyzon merolae was re-examined by electron microscopy after rapid-freeze substitution fixation to understand the minimum 
ultrastructural characteristics of eukaryotes. C. merolae cells contained one Golgi body which was composed of one to two cisternae, transfer vesicles and secretory vesicles. A trans-Golgi network was not seen, but association between the transfer vesicles and the nuclear membrane was often observed. The cells did not have a cell wall, but were covered by many filaments which were protruded from a thin electron-dense layer on the exterior surface of the cell membrane.

\section{Acknowledgments}

This research was supported by a grant (to T. Kuroiwa, No. 06101002) from the Ministry of Education, Science and Culture of Japan.

\section{References}

Allen, M. B. 1959. Studies with Cyanidium caldarium, an anomalously pigmented chlorophyte. Arch. Microbiol 32: 270-277.

Broadwater, S. T. and Scott, J. L. 1994. Ultrastructure of unicellular red algae. In Evolutiorany Pathways and Enigmatic algae: Cyanidium caldarium (Rhodophyta) and related cells. pp. 215-230. Kluwer Academic Publishers, Dordrecht.

Chapman, D. J. 1974. Taxonomic status of Cyanidium caldarium, the Porphyridiales and Goniotrichales. Nova Hedwigia Zeitschr. Kryptogamenkd. (Weinheim, lehre Braunschweig) 25(3/4): 673-682. (cytol., evol., mropholo., tax.-syst.)

De Luca, P., Taddei, R. and Varano, L. 1978. Cyanidioschyzone merolae: a new alga of thermal acidic environments. Webbia 33: $37-44$.

Giese, A. C. 1979. In Cell Physiology, pp. 1-609. W. B. Saunders Co., Philadelphia.

Kuroiwa, H. 1989. Ultrastructural examination of embryogenesis in Crepis capillaris (L.) Waller.: 1. The synergid before and after pollination. Bot. Mag. Tokyo 102: 9-24.

Kuroiwa, T., Kawazu, T., Takahashi, H., Suzuki, K., Ohta, N. and Kuroiwa, H. 1994. Comparison of ultrastructures between the ultra-small eukaryote Cyanidioschyzon merolae and Cyanidium caldarium. Cytologia 59: 149158.

-, Suzuki, K., Itoh, R., Toda, K., O’Keefe, T. C. and Kuroiwa, H. 1995. Mitochondria-dividing ring: ultrastructural basis for the mechanism of mitochondrial divison in Cyanidioschyzon merolae. Protoplasma 186: 12-23.

Merola, A., Castaldo, R., De Luca, P., Gambardella, R., Musacchio, A. and Taddei, R. 1981. Revision of Cyanidium caldarium, three species of acidiophilic algae. G. Bot. Ital. 115: 189-195.

Rambourg, A. M. and Leblond, C. P. 1967. Staining of basement membrane and associated structures by the periodic acid-Schiff and periodic acid-silver methenamine techniques. J. Ultrastr. Res. 20: 306-309.

Richardson, F. L. and Brown. T. E. 1970. Glaucosphaera vacuolata, its ultrasturcture and physiology. J. Phycol. 6: 165-171.

Suzuki, K., Ohta, L. and Kuroiwa, T. 1992. Isolation of the cell-nuclear, mitochondrial and chloroplast DNA from the ultra-small eukaryote Cyanidioschyzon merolae. Protoplasma 171: 80-84.

-, Ehara, T., Osafune, T., Kuroiwa, H., Kawano, S. and Kuroiwa, T. 1994. Behavior of mitochondria, chloroplasts and their nuclei during the mitotic cycle in the ultra-microalga Cyanidioschyzon merolae. Eur. J. Cell Biol. 63: 280-288.

-, Kawazu, T., Mita, T., Takahashi, H., Itoh, R., Toda, K. and Kuroiwa, T. 1995. Cytokinesis by a contractile ring in the primitive red alga Cyanidium caldarium RK-1. Eur. J. Cell Biol. 67: 170-178.

Takahashi, H., Takano, H., Yokoyama, A., Hara, Y., Kawano, S., Toh-e, A. and Kuroiwa, T. 1995. Isolation, characterization and chromosomal mapping of an actin gene from the primitive red alga Cyanidioschyzon merolae. Curr Genet. 28: 484-490.

Ueda, K. and Yokochi, J. 1981. Structure of Cyanidium caldarium. Bot. Mag. Tokyo 94: 159-164. 